\title{
COGNITIVE AND BEHAVIORAL EFFECTS OF TOPIRAMATE VERSUS CARBAMAZEPINE MONOTHERAPY
}

The cognitive and behavioral effects of topiramate (TPM) versus carbamazepine (CBZ) were evaluated in a multicenter, randomized, open-label, parallel-group trial at Sanggye Paik Hospital, Seoul, and other university centers in Korea. Of 112 patients of normal intelligence, ages 5 to 15 years, with benign rolandic epilepsy enrolled, 88 completed the 28 week study, 45 on TPM and 43 on CBZ. Average daily dose of TPM during the maintenance phase was $3.4 \mathrm{mg} / \mathrm{kg} / \mathrm{day}$, and that of CBZ was $21.6 \mathrm{mg} / \mathrm{kg} / \mathrm{day}$. Complete seizure control was obtained in $69.6 \%$ of TPM treated and $70.0 \%$ CBZ treated patients, and the mean percentage seizure frequency change was not different in the 2 groups. Slightly more TPM-treated patients reported memory dysfunction and somnolence, and significantly more CBZ patients reported rashes. Other adverse events were not significantly different between TPM and CBZ. Neuropsychological test results showed on the Connors teacher score, attention problems were improved with TPM and worsened by CBZ, but not significantly. In contrast, cognitive function (FSIQ, VIQ, information, block design, behavior problems) showed improved scores on CBZ and worsening for TPM, but not significantly. The maze test showed a statistically significant improvement in CBZ compared to TPMtreated patients $(\mathrm{p}=0.026)$. Cognitive measures of PIQ, digit span, picture completion, and coding, and behavioral problems of withdrawal, anxiety/depression, aggressive behavior, internalizing and externalizing problems, and delinquent behavior all showed improvement of scores for both drugs, especially for CBZ. Vocabulary, comprehension, and parent Connors scores showed worsening for both drugs, especially for TPM. (Kang H-C, Eun B-L, Lee $\mathrm{CW}$, et al. The effects on cognitive function and behavioral problems of topiramate compared to carbamazepine as monotherapy for children with benign rolandic epilepsy. Epilepsia Sept 2007;48:1716-1723). (Reprints: Dr Heung Dong Kim: E-mail: hdkimmd@yumc.yonsei.ac.kr)

COMMENT. No significant differences in cognitive and behavioral effects of TPM and CBZ were found, but scores in some tests showed a negative trend for the TPM group. Rash was a problem in $8(19 \%)$ and a reason for dropout in 4 patients taking CBZ.

\section{AUTISM AND EPILEPSY FOLLOW-UP STUDY}

One hundred thirty subjects with autism in childhood (males 104, females 26) and first seen from 1 to 14 years of age (median 4 years) were followed over 10 years, at Yokohama Central Area Habilitation Center for Children, Japan. Thirty three (25\%) developed epilepsy by age 21 years (onset at median age of 14, range 8-26), A lower cognitive level, lower social maturity score, and higher frequency of psychotropic medications were observed in patients with epilepsy vs those without. Febrile seizures occurred in the 2 groups ( $18 \%$ vs $12 \%$ ). Epileptiform EEGs were recorded in $73 \%$ of the epilepsy group vs $21 \%$ of non-epilepsy ( $<0.001$ ). EEG abnormalities occurred before the onset of epilepsy in $68 \%$ of the epilepsy group. Seizures ranged in frequency from 1 to 74 (median 3); 33\% had only 1 seizure. (Hara H. Autism and epilepsy: a retrospective follow-up study. Brain Dev Sept 2007;29:486-490). (Respond: E-mail: hara@aoitori-net.com) 